\title{
Integrated bioinformatics analysis of the osteoarthritis-associated microRNA expression signature
}

\author{
XI WANG* ${ }^{*}$ YUJIE NING ${ }^{*}$, BING ZHOU, LEI YANG, YINGTING WANG and XIONG GUO \\ School of Public Health, Xi'an Jiaotong University Health Science Center, Key Laboratory of Trace Elements and \\ Endemic Diseases, National Health and Family Planning Commission, Xi'an, Shaanxi 710061, P.R. China
}

Received February 9, 2017; Accepted August 16, 2017

DOI: $10.3892 / \mathrm{mmr} .2017 .8057$

\begin{abstract}
Numerous studies have focused on osteoarthritis (OA) cartilage injuries, with observations of abnormalities in microRNA (miRNA) expression levels. The aim of the present study was to consolidate and assess the results of these studies to identify potential miRNA biomarkers of OA. A comprehensive meta-analysis of eight independent miRNA expression studies in OA was performed using the robust rank aggregation method (RRA), which contained a total of $82 \mathrm{OA}$ and 39 normal cartilage samples. The targets of meta-signature miRNA were predicted using TargetScan v6.2, PicTar, miRDB, TarBase v7.0 and starBase databases. In addition, pathway enrichment analysis was performed to establish the biologically and functionally relevant genes involved in meta-signature miRNA regulation. Six dysregulated miRNAs were identified, including four up regulated (miR-23b-3p, miR-27b-3p, miR-211-5p and miR-16-5p) and two down regulated (miR-25-3p and miR-149-5p). The enrichment of Kyoto Encyclopedia of Genes and Genomes and Panther signaling pathways were predominantly associated with cell signaling and cell regulation. In the present study, the meta-signature miRNA identified may be used to develop a series of diagnostic and prognostic biomarkers for OA specifically for use in a clinical setting.
\end{abstract}

\section{Introduction}

Osteoarthritis (OA) is one of the most common types of arthritis and is a primary cause of disability (1). The prevalence of knee

Correspondence to: Professor Xiong Guo, School of Public Health, Xi'an Jiaotong University Health Science Center, Key Laboratory of Trace Elements and Endemic Diseases, National Health and Family Planning Commission, 76 Yanta West Road, Xi'an, Shaanxi 710061, P.R. China

E-mail: guox@xjtu.edu.cn

*Contributed equally

Key words: microRNAs, osteoarthritis, robust rank aggregation method and hip OA ranges from $20-30 \%$ in the general population worldwide (1). OA is primarily characterized by focal cartilage degradation, which may be caused by the imbalance between anabolic and catabolic factors in the joints. Furthermore, the affected joints exhibit osteophyte formation and sub-chondral bone sclerosis $(2,3)$. OA is also a multifactorial disease with strong heritability ranging from 40 to $65 \%$, which depends on the joint sites (4-7). The identification of genes associated with OA would aid with revealing the underlying molecular pathogenic mechanisms and pathways, and lead to development of the gene diagnosis and targeted therapy of OA.

Currently, the association between cartilage biology and OA in numerous studies has been concentrated on microRNAs (miRNAs). miRNA, a class of non-coding small RNAs, is widely accepted as one of the post-transcriptional modifications that influences entire intracellular molecular cascades, such as intracellular signaling $(8,9)$. Therefore, miRNAs perform critical and diverse activities in biological functions, such as apoptosis, differentiation, stem cell maintenance and metabolism. Recently, miRNA was suggested as an integral aspect of the regulatory network of chondrocyte differentiation and cartilage function, which indicates miRNA may contribute to the development of OA (10-12). The expression levels of one or a few miRNAs between OA cartilage and healthy control samples have been compared by various studies (10-12). These expression-profiling studies, which often reported conflicting results, are dispersed and have never been systematically assessed. Consequently, the potential of miRNAs serving as diagnostic markers or even therapeutic targets of OA remains unclear and requires analysis.

In the present study, a comprehensive meta-analysis was performed based on published studies, and compared miRNA expression profiles between OA cartilage and normal cartilage to overcome the limitations of these miRNA expression-profiling studies. The robust rank aggregation (RRA) method was applied in order to reduce the influences of heterogeneity (13-15) and establish the key miRNAs in OA. Various crucial miRNA target genes were predicted using bioinformatics tools. Based on this, consensus targets were combined for further analysis in corresponding databases, such as the Kyoto Encyclopedia of Genes and Genomes (KEGG) and Gene Ontology (GO) databases. The present study focused upon identifying the consistence of differently expressed miRNAs, which may provide a novel insight into the 
differentially expressed miRNA profiling studies of OA. This is considered to be of great value in improving the diagnostics, therapeutics and prognosis of OA.

\section{Materials and methods}

Literature review. A two-step literature searching strategy was used to identify the OA miRNA expression profiling studies. First, literature reviews were performed using the PubMed database (www.ncbi.nlm.nih.gov/pubmed/), Gene Expression Omnibus (www.ncbi.nlm.nih.gov/geo/) and ArrayExpress (www.ebi.ac.uk/arrayexpress) according to the following search terms: 'microRNA' OR 'miRNA' AND 'osteoarthritis' OR 'arthritis' AND 'expression' OR 'profile' OR 'profiling'. In the second step, relevant references that meet the above-mentioned criteria were carefully screened manually for further studies. The latest year of publication was 2016.

Inclusion and exclusion criteria. The following studies that: i) Profiled miRnA expression levels of patients with OA; ii) compared the expression profile of cartilage collected by surgery from OA patients with that of normal cartilage from healthy control subjects; iii) reported cut-off criteria for determining whether miRNAs were differentially expressed between the OA and normal control; and iv) reported their method for validating the profiling results were included in the meta-analysis. Therefore, miRNA profiling studies we excluded by analyzing plasma or serum sample collected from OA patients or cell lines. Review articles were also excluded.

Data extraction. Two reviewers independently extracted the following information from the included studies: Author, publication year, country of subjects, sample size and characteristics of patients, author-defined cut-off criteria of differential miRNA expression profiling, and the direction (up- or down regulated) and fold-change of the differential expression of each miRNA (if available). Subsequently, the authors aimed to reach a consensus on these items through discussion. All of the miRNA names were standardized depending on the miRBase version 21 (www.mirbase.org). miRNAs that were considered as a 'dead entry' due to re-annotation in miRBase (version 21) were omitted in subsequent meta-analysis.

RRA method for meta-analysis. The RRA method, which is a free R software package (https://cran.r-project.org/mirrors .html) was used for this meta-analysis. The gene lists of miRNAs from selected articles were ranked according to $\mathrm{P}$-values $(\mathrm{P}<0.05)$ according to the RRA method. The leave-one-out cross-validation algorithm was applied in this method. A ten thousand times repeating analysis permutation procedure was performed to calculate an average $\mathrm{P}$-value from the random gene lists, which represents the best $P$-value of each of the miRNAs. To avoid false-positive results, the Bonferroni correction of the P-values was calculated.

Prediction and filtering of dysregulated miRNAs targets. The targets of miRNA were predicted using TargetScan version 6.2 (www.targetscan.org) (16), PicTar (http://pictar.mdc-berlin.de/cgi-bin/PicTar_vertebrate.cgi) (17) and miRDB (http://www.mirdb.org/mining.html) (18). The
TarBasev7.0 database (http://diana.imis.athena-innovation. gr/DianaTools/index.php?r=tarbase/index/) (19) and starBase (http://starbase.sysu.edu.cn/targetSite.php) (20) were also used to facilitate with the screening and provide experimental proof for predicted targets. Considering the reliability, only overlapping targets predicted by more than one algorithm were selected for further investigation.

Enrichment analysis. The Database for Annotation, Visualization and Integrated Discovery (DAVID) web tool $(21,22)$ (http://david.abcc.ncifcrf.gov/) was used for pathway identification and enrichment analysis. The consensus targets of each miRNA were entered into the DAVID web tool to screen the following database Gene Ontology terms, such as KEGG, Panther and REACTOME signaling pathways.

\section{Results}

Study selection and data extraction. A total of 139 studies were identified in PubMed, and only eight of these met the inclusion criteria (Fig. 1; Table I) (23-30). The included studies were published between 2009 and 2016. Three of these were conducted with samples from Europe (United Kingdom, Greece, Spain and Germany), another two studies with samples from Asian countries (Japan and Korea) and the other two studies with samples from North America (Canada). The average number of miRNA probes was 799 (range, 157-2,100). A total of 121 human cartilage samples were used for this meta-analysis. The significant data from these studies are presented in Table I. There were 87 differentially expressed miRNAs reported in eight studies in total, out of which 46 were reported to demonstrate increased expression and 41 demonstrated decreased expression (Table I).

miRNA meta-signature. A meta-signature consisted of six dysregulated miRNAs that were significantly identified in OA samples, which included four increased and two decreased expression levels when compared with controls according to the permutation P-value. The most significantly dysregulated miRNA was miR-23b-3p, which was reported by three datasets. Two miRNAs, miR-23b-3p and miR-27b-3p were observed none cluster located at9q22.3 (Table II), and the cluster was identified in MirBase within a distance $<10 \mathrm{~kb}$. Other miRNAs were scattered on different chromosomal locations.

Target prediction of meta-signature miRNAs. The numbers of targets were presented in Fig. 2. The overlapping consensus targets of meta-signature miRNAs identified by RRA were extracted, predicted by at least two different algorithms and validated by two databases, TarBase and StarBase. miR-23b-3p and miR-16-5p were observed to have more targets than the other miRs.

Enrichment analysis for predicted target of meta-signature miRNAs. The enrichment analysis for predicted targets of meta-miRNAs was completed using the DAVID web tool. Numerous significant results were screened through the enrichment of KEGG and Panther signaling pathways, most of which were associated with cell signaling and cell regulation, as presented in Table III. 
Table I. Characteristics of studies included in the meta-analysis on microRNA expression levels in cartilage tissue samples of OA.

\begin{tabular}{|c|c|c|c|c|c|c|c|}
\hline \multirow[b]{2}{*}{ Author } & \multirow[b]{2}{*}{ Year } & \multirow[b]{2}{*}{$\begin{array}{l}\text { Samples, } \mathrm{n} \\
(\mathrm{OA} / \text { control })\end{array}$} & \multicolumn{3}{|c|}{ Differential miRNA expression } & \multirow[b]{2}{*}{ Platform } & \multirow[b]{2}{*}{ (Refs.) } \\
\hline & & & $\underset{\mathrm{n}}{\text { Upregulated, }}$ & $\begin{array}{c}\text { Downregulated, } \\
\mathrm{n}\end{array}$ & $\begin{array}{l}\text { Cut-off } \\
\text { criteria }\end{array}$ & & \\
\hline Jones et al & 2009 & $6(3 / 3)$ & 11 & 5 & $\mathrm{FC}>2.0$ & TaqmanqPCR assays & (24) \\
\hline Iliopoulos et al & 2008 & $43(33 / 10)$ & 9 & 7 & $\mathrm{FC}>1.5$ & Taqman miRNA assays & $(23)$ \\
\hline Miyaki et al & 2009 & $19(11 / 8)$ & 10 & 0 & $\mathrm{FC}>1.5$ & $\begin{array}{l}\text { mirVana miRNA } \\
\text { Labeling Kit }\end{array}$ & $(25)$ \\
\hline Diaz-Prado et al & 2012 & $10(6 / 4)$ & 1 & 6 & $\mathrm{FC}>1.5$ & miRNA PCR system & $(26)$ \\
\hline Song et al & 2013 & $15(10 / 5)$ & 7 & 11 & $\mathrm{P}<0.05$ & TaqmanqPCR assays & $(27)$ \\
\hline Li et al & 2016 & $8(4 / 4)$ & 6 & 5 & $\mathrm{FC}>1.5$ & TaqmanqPCR assays & $(28)$ \\
\hline Beyer et al & 2015 & $26(13 / 13)$ & 0 & 2 & $\mathrm{FC}>1.5$ & TaqmanqPCR assays & $(29)$ \\
\hline Nakamura et al & 2016 & $4(2 / 2)$ & 2 & 5 & $\mathrm{FC}>2.0$ & $\begin{array}{l}\text { miRCURY LNA } \\
\text { miRNA Array }\end{array}$ & $(30)$ \\
\hline Total & & $121(82 / 39)$ & 46 & 41 & & & \\
\hline
\end{tabular}

OA, osteoarthritis; FC, fold-change; qPCR, quantitative polymerase chain reaction.

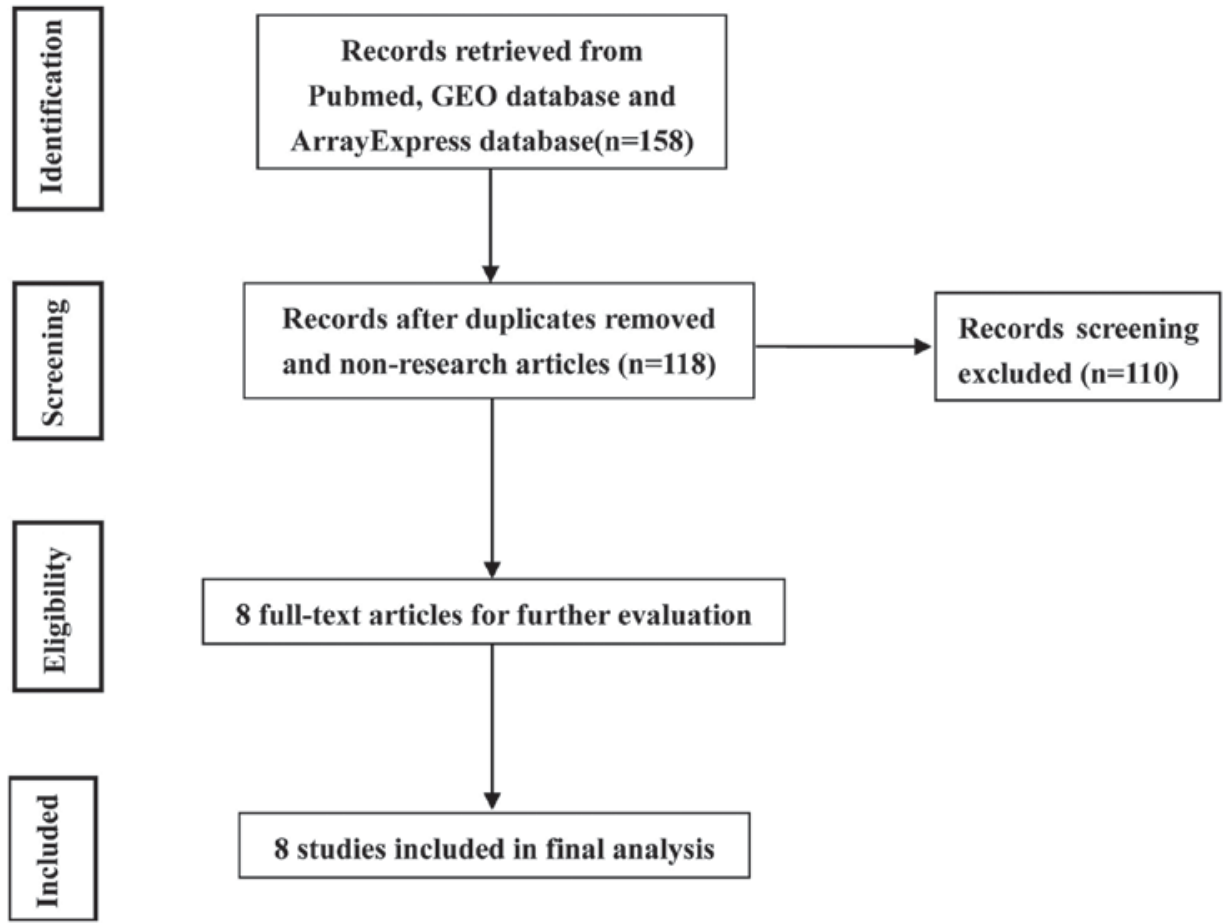

Figure 1. Flow diagram of the study selection process. GEO, Gene Expression Omnibus.

\section{Discussion}

In recent years, with the realization of the importance of miRNAs in different biological processes, numerous studies have focused on the association between miRNA expression and the development of OA. However, certain studies have shown inconsistent miRNA expression in OA cartilage samples when compared with those of healthy controls, which may be due to the following factors: i) Different platforms of profiling; ii) small sample size; and iii) inconsistent methods for data analysis. To overcome these defects, a meta-analysis using the RRA method was performed for analyzing particular miRNAs of OA from eight independent profiling experiments. When compared to the classical vote-counting method, the RRA algorithm has certain advantages, including being robust to noise, incomplete rankings, assigns scores to each element for ranking, and is efficient to compute (31). In addition, two different meta-analysis methods for differential expression of miRNAs in pancreatic ductal adenocarcinoma have been performed. In the present study, the results of 
Table II. Summary of six miRNAs that were significantly up- and downregulated in osteoarthritis cartilage tissue samples compared with normal cartilage tissue samples.

P-value

\begin{tabular}{|c|c|c|c|}
\hline iRNA & $\begin{array}{c}\text { Studies, } \\
\mathrm{n}\end{array}$ & $\begin{array}{l}\text { Robust rank } \\
\text { aggregation }\end{array}$ & Corrected \\
\hline
\end{tabular}

\begin{tabular}{|c|c|c|c|c|c|}
\hline \multicolumn{5}{|l|}{ Upregulated } & $9 \mathrm{q} 22.3$ \\
\hline miR-27b-3p & 2 & 0.000000179 & 0.020079 & 0.031 & $9 q 22.3$ \\
\hline miR-211-5p & 1 & 0.000000258 & 0.028913 & 0.01 & $15 \mathrm{q} 13.3$ \\
\hline miR-16-5p & 2 & 0.000000257 & 0.028924 & $\mathrm{P}<0.05$ & $13 q 14.2$ \\
\hline \multicolumn{6}{|l|}{ Downregulated } \\
\hline miR-25-3p & 2 & 0.000000105 & 0.011807 & 0.03 & $7 q 22.1$ \\
\hline miR-149-5p & 3 & 0.000000421 & 0.047223 & 0.000228 & $2 q 37.3$ \\
\hline
\end{tabular}

miR, microRNA.

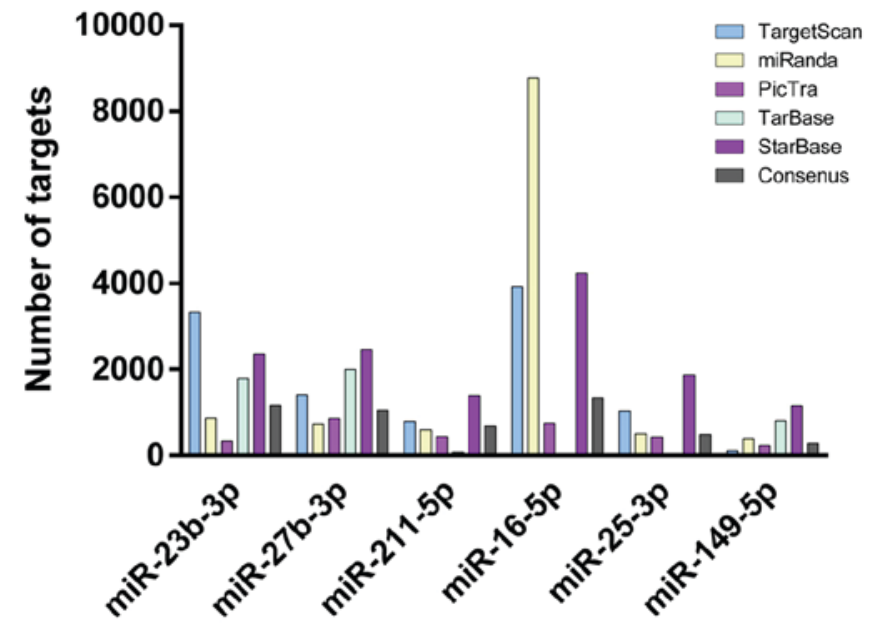

Figure 2. Target numbers of meta-signature miRs in osteoarthritis. miR, microRNA.

the different methods included the potential prognostic biomarkers, which were detected by experimental validation. Therefore, the RRA method was more accurate than the vote-counting method (32).

In the current study, six overlapping significantly dysregulated miRNAs were demonstrated with four that were upregulated and two that were downregulated. Among the identified miRNAs, miR-23b-3p demonstrated consistent up regulation in three studies. Ham et al (33) demonstrated that miR-23b-3p over expression induced chondrogenic differentiation by downregulating protein kinase A (PKA) signaling based on targeting protein kinase cAMP-activated catalytic subunit $\beta$, which encodes a catalytic subunit of PKA in synovial fluid-derived mesenchymal stem cells $(33,34)$. In 2015, Gabler et al identified that miR-23b-3p was expressed at significantly higher levels in hypertrophic chondrocytes (35). As to miR-16-5p, although the function of miR-16-5p in chondrocyte has yet to be determined, it is present at high levels in the majority of cell types and proposed to be potentially a master miRNA involved in determining mRNA stability via adenylate-uridylate-rich element sites (36). Murata et al identified that synovial fluid miR-16-5p levels of rheumatoid arthritis (RA) patients were higher than those of OA patients, and plasma miR-16-5p levels of OA patients were lower than that of healthy control subjects (37). Certain studies identified that the expression levels of miR-16-5p were decreased in the sera of early RA patients in comparison with advanced RA, which indicate miR-16-5p as a possible predictor for RA (38). miR-16-5p was reported to be over expressed in peripheral blood mononuclear cells of RA patients who were active when compared with RA patients who were relatively static, or with healthy control subjects (39). In the current meta-analysis, miR-23b-3p was significantly increased in OA cartilage tissue. These results indicate that miR-23b-3p and miR-16-5p are potential molecular markers for diagnostics, therapeutics and prognosis of OA. However, further investigation of these miRNAs in OA is considered to be necessary.

miR-149-5p was identified to be downregulated in OA chondrocytes in the study by Santini et al (40), and this decrease was correlated with increased expression levels of pro-inflammatory cytokines, such as tumor necrosis factor- $\alpha$, interleukin (IL) $-\beta$, and IL-6. Furthermore, miR-149-5p was significantly decreased in the current meta-analysis, and these results supported the hypothesis that miR-149-5p is an important regulatory molecule in the progression of OA.

The pathway enrichment analysis identified that many signaling pathways are involved in the regulation of miRNAs. Through the KEGG pathway analysis (Table III), the Wnt signaling pathway was identified to be significantly associated with development of chondrocytes, which are involved in the maintenance of fully differentiated chondrocyte phenotypes, and may, therefore, be crucial in cartilage homeostasis throughout life (41).

There are certain limitations associated with the meta-analysis on miRNA expression profiles. The included studies depended on a variety of microarray platforms and validation criteria. Hence, it is important to apply a more 
Table III. GO process, KEGG pathway, Panther pathway and REACTOME pathway enriched by meta-signature miRNA targets.

Pathway enrichment analysis

GO process

GO:0044260: Cellular macromolecule metabolic process

GO:0043170: Macromolecule metabolic process

GO:0006464: Protein modification process

GO:0043687: Post-translational protein modification

GO:0043412: Biopolymer modification

GO:0044267: Cellular protein metabolic process

GO:0044237: Cellular metabolic process

GO:0009987: Cellular process

GO:0060255: Regulation of macromolecule metabolic process

GO:0080090: Regulation of primary metabolic process

GO:0019222: Regulation of metabolic process

KEGG pathway

04115: p53 signaling pathway

05215: Prostate cancer

05200: Pathways in cancer

05210: Colorectal cancer

05222: Small cell lung cancer

00562: Inositol phosphate metabolism

04110: Cell cycle

04070: Phosphatidylinositol signaling system

04310: Wnt signaling pathway

04120: Ubiquitin mediated proteolysis

Panther pathway

P00030: Hypoxia response via hypoxia-inducible factor activation Targets

P-value

286

290

110

94

111

149

315

440

184

183

196

10

11

23

10

9

7

11

8

12

11
P00034: Integrin signalling pathway

P00033: Insulin/insulin-like growth factor pathway-protein kinase B signaling cascade

P04398: p53 pathway feedback loops 2

P00048: Phosphoinositide 3 kinase pathway

P00046: Oxidative stress response

P00052: TGF- $\beta$ signaling pathway

REACTOME pathway

REACT_12034: Signaling by bone morphogenetic proteins

REACT_6844: Signaling by TGF- $\beta$
8.64962E-2

$1.05878 \mathrm{E}-2$

$2.70971 \mathrm{E}-2$

$2.16068 \mathrm{E}-2$

$2.96494 \mathrm{E}-2$

$5.41348 \mathrm{E}-2$

$3.20433 \mathrm{E}-2$

$8.45760 \mathrm{E}-2$

9.34049E-2

4.36152E-2

$6.01876 \mathrm{E}-2$

4.92024E-4

$9.08174 \mathrm{E}-4$

$1.91181 \mathrm{E}-3$

$2.28645 \mathrm{E}-3$

8.12637E-3

$1.02755 \mathrm{E}-2$

$1.08581 \mathrm{E}-2$

$1.34821 \mathrm{E}-2$

$1.49651 \mathrm{E}-2$

1.96911E-2

1.28214E-2

$1.50999 \mathrm{E}-2$

$2.58629 \mathrm{E}-2$

3.93284E-2

4.35708E-2

4.49204E-2

4.59938E-2

3.08411E-2

4.39359E-2

GO, Gene Ontology; KEGG, Kyoto Encyclopedia of Genes and Genomes; TGF, transforming growth factor.

objective and comprehensive methodological standardization for maximum reliability. Three of the included studies in the current meta-analysis involved Asian subjects, three involved European subjects and the other two involved North America subjects, which could explain the inconsistency of miRNA expression directions. Therefore, future studies that validate profiling results in the same ethnicities may provide improved results. In addition, certain studies did not confirm whether their microarray results were in vitro or in vivo, nor did they assess the prognostic association between miRNAs and OA, which is important to validate OA biomarkers. Furthermore, the inconsistent profiling results of miRNA expression maybe due to various potential reasons, including differences in samples, the genetic and environmental background of tissue donors, the clinical pathological characteristics of tissue donors, and the expression profiling platforms.

In conclusion, six significant dysregulated miRNAs were identified across eight independent studies in OA. The meta-signature miRNAs and associated pathways may present as promising markers for clinical intervention. Further investigations should focus upon the molecular mechanisms that miRNAs may exert in the occurrence and progression of OA.

\section{Acknowledgements}

This study was funded by grants from the National Natural Science Foundation of China (grant no. 81472924). 


\section{References}

1. Taylor AM: Metabolic and endocrine diseases, cartilage calcification and arthritis. Curr Opin Rheumatol 25: 198-203, 2013.

2. Bian Q, Wang YJ, Liu SF and Li YP: Osteoarthritis: Genetic factors, animal models, mechanisms, and therapies. Front Biosci (Elite Ed) 4: 74-100, 2012.

3. Loeser RF, Goldring SR, Scanzello CR and Goldring MB: Osteoarthritis: A disease of the joint as an organ. Arthritis Rheum 64: 1697-1707, 2012.

4. Cicuttini FM and Spector TD: Genetics of osteoarthritis. Ann Rheum Dis 55: 665-667, 1996.

5. Spector TD, Cicuttini F, Baker J, Loughlin J and Hart D: Genetic influences on osteoarthritis in women: A twin study. BMJ 312: 940-943, 1996

6. Kraus VB, Jordan JM, Doherty M, Wilson AG, Moskowitz R, Hochberg M, Loeser R, Hooper M, Renner JB, Crane MM, et al The genetics of generalized osteoarthritis (GOGO) study: Study design and evaluation of osteoarthritis phenotypes. Osteoarthritis Cartilage 15: 120-127, 2007.

7. Tsezou A: Osteoarthritis year in review 2014: Genetics and genomics. Osteoarthritis Cartilage 22: 2017-2024, 2014.

8. Berezikov E: Evolution of microRNA diversity and regulation in animals. Nat Rev Genet 12: 846-860, 2011.

9. Jingsheng S, Yibing W, Jun X, Siqun W, Jianguo W, Feiyan C, Gangyong $\mathrm{H}$ and Jie $\mathrm{C}$ : MicroRNAs are potential prognostic and therapeutic targets in diabetic osteoarthritis. J Bone Miner Metab 33: 1-8, 2015.

10. Baxter D, McInnes IB and Kurowska-Stolarska M: Novel regulatory mechanisms in inflammatory arthritis: A role for microRNA. Immunol Cell Biol 90: 288-292, 2012.

11. Hong E and Reddi AH: MicroRNAs in chondrogenesis, articular cartilage, and osteoarthritis: Implications for tissue engineering. Tissue Eng Part B Rev 18: 445-453, 2012.

12. Le LT, Swingler TE and Clark IM: Review: The role of microRNAs in osteoarthritis and chondrogenesis. Arthritis Rheum 65: 1963-1974, 2013.

13. Griffith OL, Melck A, Jones SJ and Wiseman SM: Meta-analysis and meta-review of thyroid cancer gene expression profiling studies identifies important diagnostic biomarkers. J Clin Oncol 24: 5043-5051, 2006.

14. Guan P, Yin Z, Li X, Wu W and Zhou B: Meta-analysis of human lung cancer microRNA expression profiling studies comparing cancer tissues with normal tissues. J Exp Clin Cancer Res 31: $54,2012$.

15. Li MY and Hu XX: Meta-analysis of microRNA expression profiling studies in human cervical cancer. Med Oncol 32: 510, 2015.

16. Grimson A, Farh KK, Johnston WK, Garrett-Engele P, Lim LP and Bartel DP: MicroRNA targeting specificity in mammals: Determinants beyond seed pairing. Mol Cell 27: 91-105, 2007.

17. Blin K, Dieterich C, Wurmus R, Rajewsky N, Landthaler M and Akalin A: DoRiNA 2.0-upgrading the doRiNA database of RNA interactions in post-transcriptional regulation. Nucleic Acids Res 43 (Database issue): D160-D167, 2015.

18. Wong N and Wang X: miRDB: An online resource for microRNA target prediction and functional annotations. Nucleic Acids Res 43 (Database issue): D146-D152, 2015.

19. Vlachos IS, Paraskevopoulou MD, Karagkouni D, Georgakilas G, Vergoulis T, Kanellos I, Anastasopoulos IL, Maniou S, Karathanou K, Kalfakakou D, et al: DIANA-TarBase v7.0: Indexing more than half a million experimentally supported miRNA:mRNA interactions. Nucleic Acids Res 43 (Database issue): D153-D159, 2015.

20. Yang JH, Li JH, Shao P, Zhou H, Chen YQ and Qu LH: starBase: A database for exploring microRNA-mRNA interaction maps from Argonaute CLIP-Seq and Degradome-Seq data. Nucleic Acids Res 39 (Database issue): D202-D209, 2011.

21. Huang da W, Sherman BT and Lempicki RA: Bioinformatics enrichment tools: Paths toward the comprehensive functional analysis of large gene lists. Nucleic Acids Res 37: 1D-13D, 2009.

22. Huang da W, Sherman BT and Lempicki RA: Systematic and integrative analysis of large gene lists using DAVID bioinformatics resources. Nat Protoc 4: 44-57, 2009.
23. Iliopoulos D, Malizos KN, Oikonomou P and Tsezou A: Integrative microRNA and proteomic approaches identify novel osteoarthritis genes and their collaborative metabolic and inflammatory networks. PLoS One 3: e3740, 2008.

24. Jones SW, Watkins G, Le Good N, Roberts S, Murphy CL, Brockbank SM, Needham MR, Read SJ and Newham P: The identification of differentially expressed microRNA in osteoarthritic tissue that modulate the production of TNF-alpha and MMP13. Osteoarthritis Cartilage 17: 464-472, 2009.

25. Miyaki S, Nakasa T, Otsuki S, Grogan SP, Higashiyama R, Inoue A, Kato Y, Sato T, Lotz MK and Asahara H: MicroRNA-140 is expressed in differentiated human articular chondrocytes and modulates interleukin-1 responses. Arthritis Rheum 60: 2723-2730, 2009.

26. Diaz-Prado S, Cicione C, Muiños-López E, Hermida-Gómez T, Oreiro N, Fernández-López C and Blanco FJ: Characterization of microRNA expression profiles in normal and osteoarthritic human chondrocytes. BMC Musculoskelet Disord 13: 144, 2012.

27. Song J, Kim D, Lee CH, Lee MS, Chun CH and Jin EJ: MicroRNA-488 regulates zinc transporter SLC39A8/ZIP8 during pathogenesis of osteoarthritis. J Biomed Sci 20: 31, 2013.

28. Li YH, Tavallaee G, Tokar T, Nakamura A, Sundararajan K, Weston A, Sharma A, Mahomed NN, Gandhi R, Jurisica I and Kapoor M: Identification of synovial fluid microRNA signature in knee osteoarthritis: Differentiating early- and late-stage knee osteoarthritis. Osteoarthritis Cartilage 24: 1577-1586, 2016.

29. Beyer C,Zampetaki A, Lin NY, Kleyer A, Perricone C, Iagnocco A, Distler A, Langley SR, Gelse K, Sesselmann S, et al: Signature of circulating microRNAs in osteoarthritis. Ann Rheum Dis 74: e18, 2015.

30. Nakamura A, Rampersaud YR, Sharma A, Lewis SJ, Wu B, Datta P, Sundararajan K, Endisha H, Rossomacha E, Rockel JS, et al: Identification of microRNA-181a-5p and microRNA-4454 as mediators of facet cartilage degeneration. JCI Insight 1: e86820, 2016

31. Kolde R, Laur S, Adler P and Vilo J: Robust rank aggregation for gene list integration and meta-analysis. Bioinformatics 28: 573-580, 2012.

32. Ma MZ, Kong X, Weng MZ, Cheng K, Gong W, Quan ZW and Peng CH: Candidate microRNA biomarkers of pancreatic ductal adenocarcinoma: Meta-analysis, experimental validation and clinical significance. J Exp Clin Cancer Res 32: 71, 2013.

33. Ham O, Lee CY, Song BW, Lee SY, Kim R, Park JH, Lee J, Seo HH, Lee CY, Chung YA, et al: Upregulation of miR-23b enhances the autologous therapeutic potential for degenerative arthritis by targeting PRKACB in synovial fluid-derived mesenchymal stem cells from patients. Mol Cells 37: 449-456, 2014.

34. Mirzamohammadi F, Papaioannou G and Kobayashi T: MicroRNAs in cartilage development, homeostasis, and disease. Curr Osteoporos Rep 12: 410-419, 2014

35. Gabler J, Ruetze M, Kynast KL, Grossner T, Diederichs S and Richter W: Stage-Specific miRs in chondrocyte maturation: Differentiation-dependent and hypertrophy-related miR clusters and the miR-181 family. Tissue Eng Part A 21: 2840-2851, 2015.

36. Asirvatham AJ, Magner WJ and Tomasi TB: miRNA regulation of cytokine genes. Cytokine 45: 58-69, 2009.

37. Murata K, Yoshitomi H, Tanida S, Ishikawa M, Nishitani K, Ito $\mathrm{H}$ and Nakamura T: Plasma and synovial fluid microRNAs as potential biomarkers of rheumatoid arthritis and osteoarthritis. Arthritis Res Ther 12: R86, 2010

38. Filková M, Aradi B, Senolt L, Ospelt C, Vettori S, Mann H, Filer A, Raza K, Buckley CD, Snow M, et al: Association of circulating miR-223 and miR-16 with disease activity in patients with early rheumatoid arthritis. Ann Rheum Dis 73: 1898-1904, 2014.

39. Pauley KM, Satoh M, Chan AL, Bubb MR, Reeves WH and Chan EK: Upregulated miR-146a expression in peripheral blood mononuclear cells from rheumatoid arthritis patients. Arthritis Res Ther 10: R101, 2008.

40. Santini P, Politi L, Vedova PD, Scandurra R and Scotto d'Abusco A: The inflammatory circuitry of miR-149 as a pathological mechanism in osteoarthritis. Rheumatol Int 34: 711-716, 2014.

41. Ma B, Landman EB, Miclea RL, Wit JM, Robanus-Maandag EC, Post JN and Karperien M: WNT signaling and cartilage: Of mice and men. Calcif Tissue Int 92: 399-411, 2013. 